\title{
Structural health monitoring and damage detection of an arch bridge using a minimum mean square error estimator
}

\author{
J. Kullaa \\ Helsinki Metropolia University of Applied Sciences, Helsinki, Finland \\ F. Magalhães \& Á. Cunha \\ ViBest-CONSTRUCT, Faculty of Engineering, University of Porto, Porto, Portugal
}

\begin{abstract}
A concrete arch bridge in the city of Porto is equipped with a monitoring system continuously recording structural vibrations. The vibration characteristics are extracted from the measurements using automatic output-only identification. Statistical deviation of the natural frequencies of the structure can be used as an indication of damage. Since the variable environmental and operational conditions also affect these features, a minimum mean square error estimation algorithm was used to eliminate their influences from the data. The measurement of the environmental variables (e.g. temperature) is not necessary. During two years, no damage emerged, which is in agreement with the damage detection results. For damage simulation, a constant frequency shift, quantified by means of a numerical model, was added to a set of experimental samples. After the artificial introduction of damage, the extreme value statistics control charts showed an abrupt and permanent change in the data, from which damage could be detected.
\end{abstract}

\section{INTRODUCTION}

Ageing of civil infrastructure is a major concern both for economic and safety reasons. In order to extend the life of ageing infrastructure or to design new intelligent structures, structural health monitoring (SHM) can be applied to estimate the state of the structure and to get an early warning of damage.

Continuous monitoring of structures using a permanently installed vibration sensor network can complement or partially replace visual inspection. With an early detection of damage, structures can be repaired at lower costs before more serious deterioration occurs.

Damage detection can be based on vibration measurements tracking changes of dynamic characteristics of the structure. The dynamic characteristics are extracted from measured structural response, e.g. acceleration or strain histories. In this study, the dynamic characteristics are the lowest natural frequencies of the structure. Because their values vary between measurements, statistical analysis is needed to separate damage from normal variability.

It is well known that environmental or operational variations also have an influence on the vibration characteristics of structures (Wahab \& De Roeck 1997; Farrar et al. 1997; Alampalli 1998; Cornwell et al. 1999; Peeters \& De Roeck 2000; Rohrmann et al. 2000; Darbre \& Proulx 2001). Due to environmental effects, such as temperature variation, the variability of the natural frequencies can be so large that early detection of damage becomes difficult. Therefore, special attention must be given to mitigate the environmental effects on the data.

It is possible to minimize the environmental or operational influences using methods in which the measurement of the underlying variables is not necessary (Kullaa 2002, 2003, 2004, 2005, 2006, 2012, 2014; Manson 2002; Sohn et al. 2003; Vanlanduit et al. 2005; Yan et al. 2005a, b; Basseville et al. 2006; Yan \& Golinval 2006; Lämsä \& Kullaa 2007; Gorinevsky et al. 2007).

It is also possible to utilize the measured environmental variables. This has been done successfully by Magalhães et al. (2011). However, sometimes not all underlying variables are available or the relationship between the environmental variables and the dynamic properties of the structure is complex or non-linear. Then, damage detection from the structural response alone could be more practical and automatic (Kullaa 2011).

The paper is organized as follows. The method for data analysis for damage detection under environmental influences is presented in Section 2. The structural health monitoring system with data acquisition and system identification is described in Section 3. Section 4 shows the results of damage detection using both the original data from the undamaged structure and the same data simulating damage with a shift in the natural frequencies. Finally, concluding remarks are given in Section 5. 


\section{DAMAGE DETECTION}

\subsection{Environmental effects}

In data-based SHM, training data are first acquired from the undamaged structure. Then, each new measurement is compared against the training data for statistically significant changes in the dynamic characteristics, such as natural frequencies of the structure. However, the normal variability of the natural frequencies due to environmental effects is often higher than the changes due to damage. Therefore, removing the environmental effects from the data is crucial for reliable and sensitive statistical analysis for damage detection.

It is assumed that the number of extracted features is higher than the number of environmental variables. In that case, correlation between the natural frequencies can be utilized to minimize the environmental effects and the measurement of the environmental variables is not necessary.

The idea is illustrated in Figure 1 with a joint distribution of two variables $x_{1}$ and $x_{2}$. The variability of either variable alone is relatively large (black dashed lines). However, this variability can be decreased utilizing the correlation between them. Using the conditional distributions $p\left(x_{1} \mid x_{2}\right)$ and $p\left(x_{2} \mid x_{1}\right)$ instead, the residuals will be smaller. The conditional distributions are shown in red in Figure 1 for a sample data point shown with a red circle.

If the environmental effects are not taken into account, the residuals are higher and small damage can remain undetected. Also seasonal effects can make detection difficult.

The conditional distributions can be obtained from the data covariance matrix as shown in the following (Bishop 2006, Kullaa 2011).

With enough redundancy, a subset of observations $\mathbf{x}$ can be estimated using the remaining variables. Each observation is divided into observed variables $\mathbf{v}$ and missing variables $\mathbf{u}$ :

$$
\mathbf{x}=\left\{\begin{array}{l}
\mathbf{u} \\
\mathbf{v}
\end{array}\right\}
$$

with a partitioned mean vector $\mu$ and data covariance matrix $\Sigma$ :

$$
\boldsymbol{\mu}=\left\{\begin{array}{l}
\boldsymbol{\mu}_{u} \\
\boldsymbol{\mu}_{v}
\end{array}\right\}, \quad \boldsymbol{\Sigma}=\left[\begin{array}{ll}
\boldsymbol{\Sigma}_{u u} & \boldsymbol{\Sigma}_{u v} \\
\boldsymbol{\Sigma}_{v u} & \boldsymbol{\Sigma}_{v v}
\end{array}\right]=\left[\begin{array}{ll}
\boldsymbol{\Gamma}_{u u} & \boldsymbol{\Gamma}_{u v} \\
\boldsymbol{\Gamma}_{v u} & \boldsymbol{\Gamma}_{v v}
\end{array}\right]^{-1}
$$

where the precision matrix $\Gamma$ is defined as the inverse of the covariance matrix $\Sigma$ and is also written in the partitioned form. A linear minimum mean square error (MMSE) estimate for $\mathbf{u} \mid \mathbf{v}(\mathbf{u}$ given $\mathbf{v}$ ) is obtained by minimizing the mean-square error (MSE) and can be computed either using the covariance or precision matrix. If each variable is estimated in turn, the formulas based on the precision ma- trix result in a more efficient algorithm (Kullaa 2010). The expected value of the missing variable is:

$\hat{\mathbf{u}}=E(\mathbf{u} \mid \mathbf{v})=\boldsymbol{\mu}_{u}-\boldsymbol{\Gamma}_{u u}^{-1} \boldsymbol{\Gamma}_{u v}\left(\mathbf{v}-\boldsymbol{\mu}_{v}\right)$.

and the covariance of the conditional distribution is

$\operatorname{cov}(\mathbf{u} \mid \mathbf{v})=\Gamma_{u u}^{-1}$

The residuals for each variable are generated as follows.

$\boldsymbol{\varepsilon}=\mathbf{u}-\hat{\mathbf{u}}$

All residuals are standardized according to the training data. These standardized residuals are then subjected to principal component analysis (PCA). In PCA, all samples, training and test data, are included in order to find the direction in the data space with the largest change, which is supposed to indicate damage. A control chart (Montgomery 1997) is designed for the principal component scores of the residuals. The in-control samples in the control chart may be the same as the training data or they can be selected differently from other measurements from the undamaged structure.

\subsection{Algorithm:}

An algorithm is proposed to mitigate the environmental effects for more reliable damage detection. The algorithm consists of the following functions.

(1) MMSE estimation to estimate one variable in turn using the remaining variables (Eq. 3)

(2) Residual generation by subtracting the estimate from the actual measurement (Eq. 5)

(3) Data standardization so that all residuals have zero mean and unit variance for the training data

(4) Applying PCA to all data (residuals) and reduce dimensionality by selecting the first PC scores of the residuals in the direction of maximum change (damage)

(5) Designing a control chart for the PC scores to detect damage. Control chart is designed using in-control samples (undamaged). 


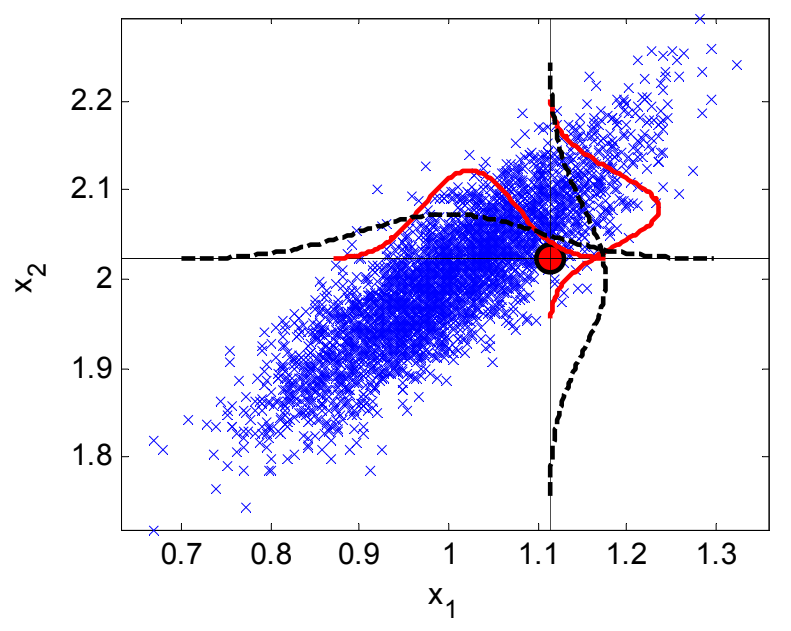

Figure 1. Illustrative example of a joint distribution of two variables. Conditional distributions are shown in red for a data point (red circle). The marginal distributions are shown in black dashed lines.

\section{BRIDGE AND MONITORING SYSTEM}

The Infante D. Henrique Bridge, over the Douro River, links the cities of Porto and Gaia, located in the north of Portugal. The bridge is composed of a rigid prestressed concrete box girder, with a height of $4.50 \mathrm{~m}$, supported by a shallow and thin reinforced concrete arch, with a thickness of $1.50 \mathrm{~m}$ (Fig. 2). The arch spans $280 \mathrm{~m}$ between abutments and rises $25 \mathrm{~m}$ until the crown (Adão da Fonseca \& Millanes Matos 2005).

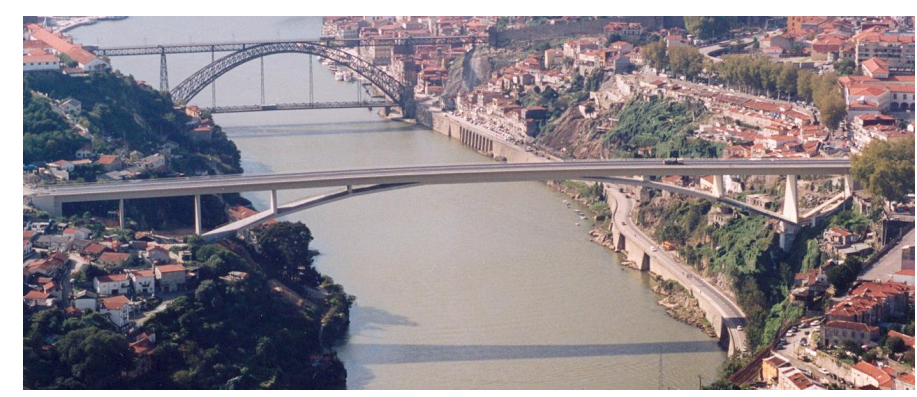

Figure 2. Infante D. Henrique Bridge in the foreground and Luiz I Bridge in the background.

The installed dynamic monitoring system is essentially composed of two digitizers that receive the signals from 12 force-balanced accelerometers, which are installed inside the deck box girder and distributed along the bridge according to the scheme presented in Figure 3. In order to obtain a good characterization of the lateral bending, vertical bending and torsion modes, each section has three sensors: one measures lateral acceleration and the other two measure vertical acceleration at the downstream and upstream sides.

The data produced by the two digitizers are continuously transferred to FEUP as ASCII files, con- taining acceleration time series with a measurement period of 30 minutes and a sampling ratio of $50 \mathrm{~Hz}$ (Magalhães et al. 2008).

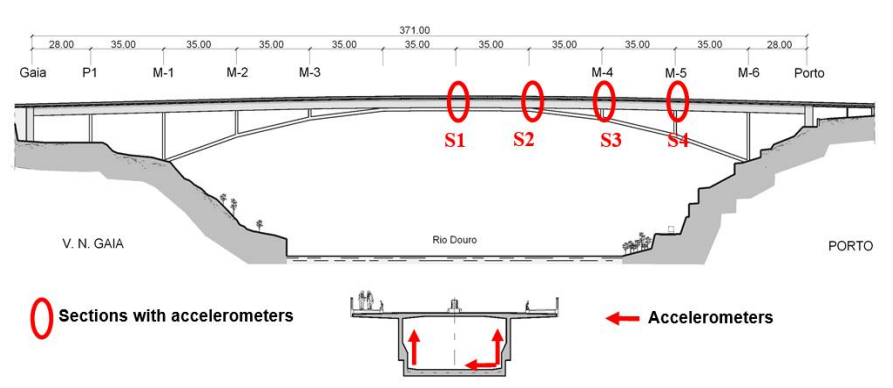

Figure 3. Dynamic Monitoring system: distribution of the accelerometers.

The acquired data are continuously processed with a dynamic monitoring software DynaMo, which includes routines for online, automatic identification of the modal parameters of the bridge (Magalhães et al. 2009).

Previous analyses of the acquired database are presented in Magalhães et al. (2011) and Magalhães et al. (2014).

\section{RESULTS}

The data consist of 12 identified natural frequencies. In some samples, one or more frequencies were not identified and those entries were replaced with $\mathrm{NaN}$ (not-a-number) in the data matrix. Two approaches were studied: (1) removing all samples including one or more missing data, and (2) replacing the missing data with estimated data. The results from both approaches were similar. Here, option (1) is only presented. Estimation of missing data has been applied in Bocca et al. (2011).

The training data are the measurements in the first year. The training data are also used as the incontrol data to design the control charts.

\subsection{No damage}

First, the original data with no damage were analysed. The identified 12 lowest natural frequencies are plotted in Figure 4, in which seasonal variability can be observed.

Comparison of residuals for mode 5 is shown in Figure 5 from two different distributions: marginal distribution (top) and conditional distribution (bottom). It can be seen that the residual of the conditional distribution is smaller than that of the marginal distribution. Also, the seasonal variability cannot be observed in the conditional distribution.

The data for damage detection are the first principal component scores of the residuals of the two year data. If these scores are not normally distributed 
and the distribution is unknown, a generalized extreme value distribution can be used independently of the data distribution (Castillo 1988, Worden et al. 2002). The data are divided into subgroups (here of size $n=50$ ) and the subgroup minima and maxima are recorded. Extreme value distributions are identified for both the subgroup minima and maxima of the in-control samples (training data). The control limits are computed to these distributions by choosing the probability of exceedance (here 0.001). The extreme values of all data are plotted on the control chart to see if the test samples exceed the control limits indicating damage.

The extreme value statistics (EVS) control charts for the first principal component of the residuals of the two year data are plotted using the marginal distributions (Fig. 6) and the conditional distributions (Fig. 7).

If the environmental variability is not taken into account in the data analysis, the first PC scores exhibit seasonal variability, which is shown in Figure 6. Functions (1) and (2) in Section 2.2 were omitted in the data analysis. It can be seen that damage detection is difficult, or even impossible, if environmental effects are not taken into account.

On the other hand, although Figure 7 indicates occasional false alarms, no permanent change can be seen. Because it is known that the data were measured from the undamaged structure, it can be concluded that damage detection worked fine.

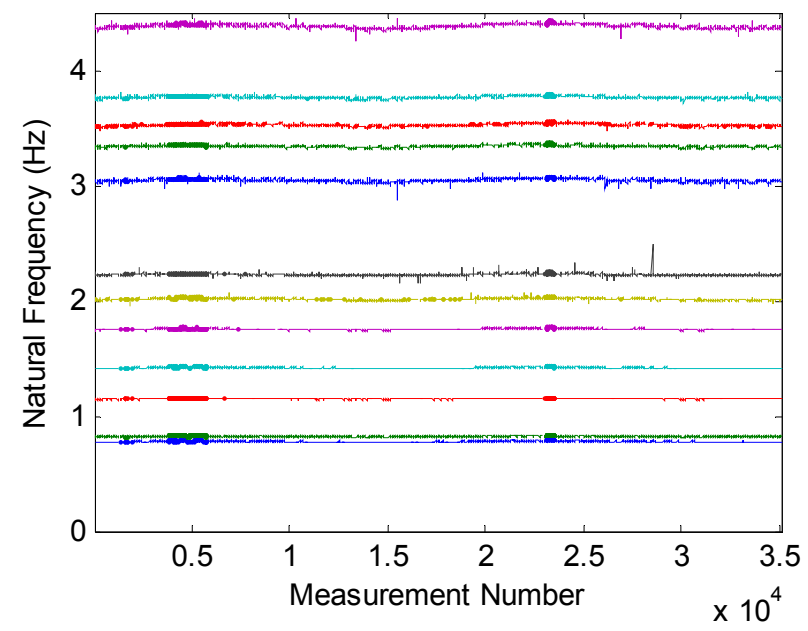

Figure 4. Identified 12 natural frequencies from each 30minutes measurement during two years.
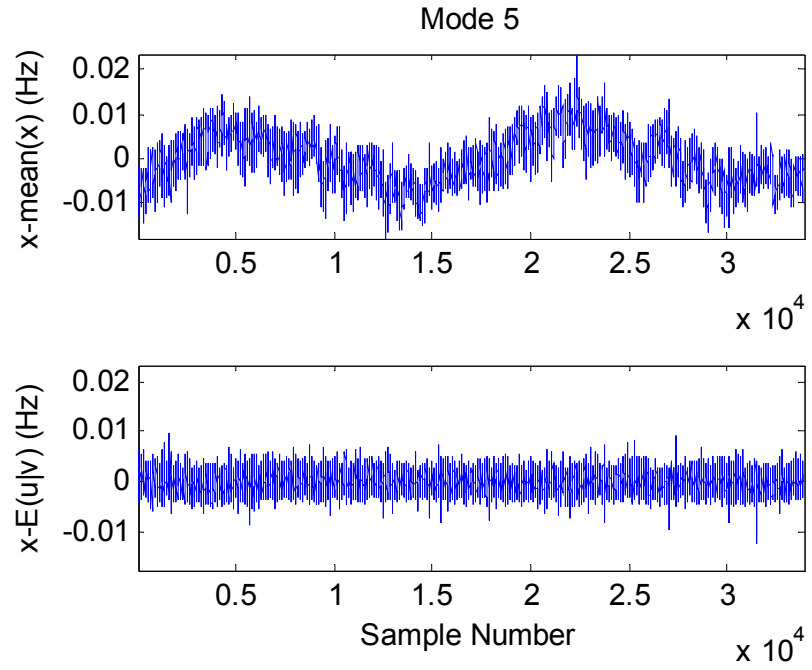

Figure 5. Residuals of the natural frequency of mode 5. Top: data mean subtracted; bottom: conditional mean subtracted.

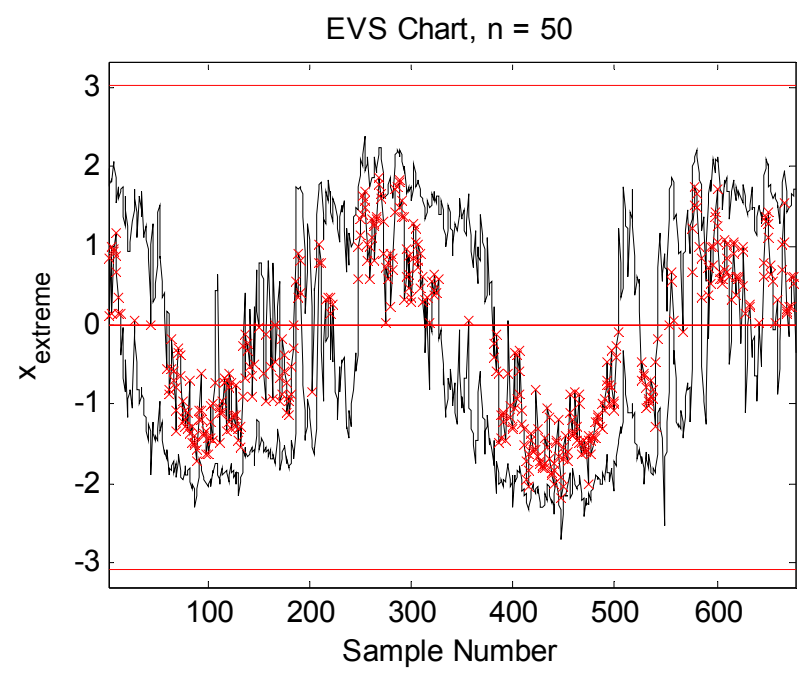

Figure 6. EVS chart of the first PC scores of the residual using the raw frequency data where the environmental effects were not removed.

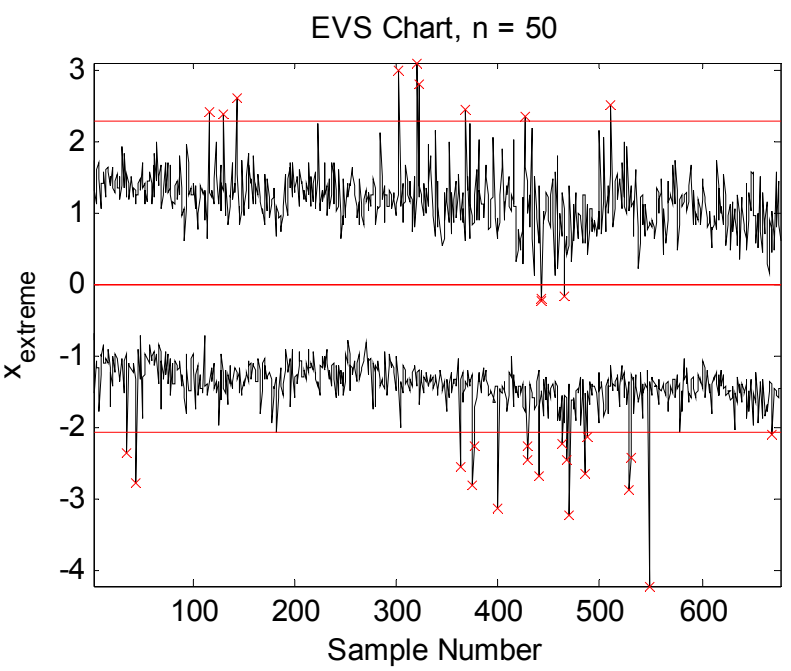

Figure 7. The first PC scores of the residuals using the conditional distributions of the frequency data where the environmental effects were removed. 


\subsection{Damage simulation}

The bridge did not indicate damage during the two years of monitoring. In order to study the damage detection capability of the proposed method, changes in natural frequencies due to damage had to be simulated.

The likely consequences of extreme events or structural ageing were modelled in a simplified manner by reductions of the bending stiffness in small segments of selected bridge components using a finite element model tuned with the experimentally identified modal parameters of the bridge. Figure 8 shows the location and the extent of the four studied damage scenarios. All damage scenarios were modelled by a reduction of the vertical bending stiffness of $10 \%$ over the length marked in the figure: $D 1-$ $8.75 \mathrm{~m} ; D 2-10.0 \mathrm{~m} ; D 3-3.0 \mathrm{~m}$ and $D 4-5.0 \mathrm{~m}$ (Magalhães et al. 2011).

The numerical simulations resulted in natural frequencies corresponding to the experimentally identified frequencies. Absolute frequencies were, however, not used, because their difference to the experimental values was too large, larger than the effects of damage. Instead, the introduction of damage in the experimental values was based on the natural frequency shifts between the numerical models of the damaged and undamaged structure. These frequency shifts were then added to the experimentally identified frequencies to simulate damage.

Table 1 shows the mean value and standard deviation of each 12 natural frequency during the two years' monitoring period. The statistics were computed from the identified frequencies of the undamaged structure. Also shown are the standard deviations of the conditional distributions, which are smaller due to the removal of the environmental influences.

The impact of each damage scenario on the 12 natural frequencies is quantified in Tables 2 and 3. Bending modes were only affected due to the characteristics of the damage scenarios. The tables show the absolute frequency shifts and the changes relative to the standard deviations.

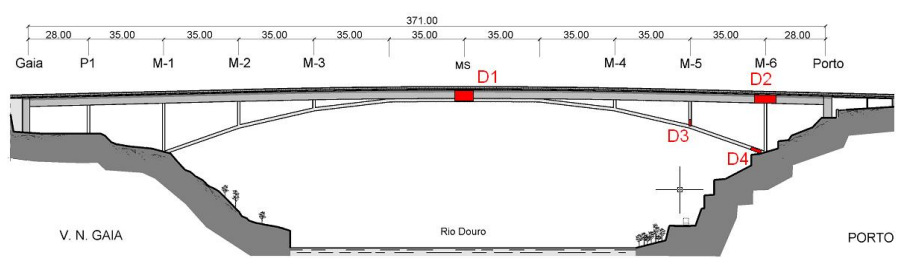

Figure 8. Location and extent of the simulated damage scenarios $D 1-D 4$.
Table 1. For each 12 natural frequency: mean value, standard deviation, and standard deviation of the conditional distribution.

\begin{tabular}{llll}
\hline No & $\begin{array}{l}f_{n} \\
\mathrm{~Hz}\end{array}$ & $\begin{array}{l}\sigma_{f} \\
\mathrm{mHz}\end{array}$ & $\begin{array}{l}\sigma_{u \mid v} \\
\mathrm{mHz}\end{array}$ \\
\hline 1 & 0.7781 & 2.07 & 0.80 \\
2 & 0.8211 & 4.22 & 2.31 \\
3 & 1.1466 & 2.67 & 1.08 \\
4 & 1.4156 & 4.48 & 1.49 \\
5 & 1.7518 & 5.28 & 1.59 \\
6 & 2.0121 & 7.86 & 2.25 \\
7 & 2.2253 & 6.47 & 3.43 \\
8 & 3.0420 & 11.81 & 4.03 \\
9 & 3.3385 & 9.07 & 3.71 \\
10 & 3.5237 & 10.47 & 4.83 \\
11 & 3.7616 & 10.21 & 3.80 \\
12 & 4.3807 & 15.72 & 5.15 \\
\hline
\end{tabular}

Table 2. Frequency shifts due to damage $D 1$ and $D 2$ : absolute shift and shift relative to either standard deviation.

\begin{tabular}{|c|c|c|c|c|c|c|}
\hline \multirow[t]{2}{*}{ No } & \multicolumn{3}{|l|}{$D 1$} & \multicolumn{3}{|l|}{$D 2$} \\
\hline & $\begin{array}{l}\overline{\Delta f_{n}} \\
\mathrm{mHz}\end{array}$ & $\Delta f_{n} / \sigma_{f}$ & $\Delta f_{n} / \sigma_{u \mid v}$ & $\begin{array}{l}\overline{\Delta f_{n}} \\
\mathrm{mHz}\end{array}$ & $\Delta f_{n} / \sigma_{f}$ & $\Delta f_{n} / \sigma_{u \mid v}$ \\
\hline 1 & 0.00 & 0.00 & 0.00 & 0.00 & 0.00 & 0.00 \\
\hline 2 & 0.00 & 0.00 & 0.00 & -1.60 & -0.38 & -0.69 \\
\hline 3 & -1.30 & -0.49 & -1.20 & -0.30 & -0.11 & -0.28 \\
\hline 4 & -1.80 & -0.40 & -1.21 & -2.30 & -0.51 & -1.55 \\
\hline 5 & 0.00 & 0.00 & 0.00 & 0.00 & 0.00 & 0.00 \\
\hline 6 & -0.10 & -0.01 & -0.04 & -3.00 & -0.38 & -1.33 \\
\hline 7 & 0.80 & 0.12 & 0.23 & 0.00 & 0.00 & 0.00 \\
\hline 8 & -4.10 & -0.35 & -1.02 & -2.40 & -0.20 & -0.59 \\
\hline 9 & 0.30 & 0.03 & 0.08 & 0.00 & 0.00 & 0.00 \\
\hline 10 & 0.00 & 0.00 & 0.00 & -0.30 & -0.03 & -0.06 \\
\hline 11 & 0.00 & 0.00 & 0.00 & 0.00 & 0.00 & 0.00 \\
\hline 12 & -0.10 & -0.01 & -0.02 & -0.30 & -0.02 & -0.06 \\
\hline
\end{tabular}

Table 3. Frequency shifts due to damage $D 3$ and $D 4$ : absolute shift and shift relative to either standard deviation.

\begin{tabular}{|c|c|c|c|c|c|c|}
\hline \multirow[t]{2}{*}{ No } & \multicolumn{3}{|l|}{ D3 } & \multicolumn{3}{|l|}{$D 4$} \\
\hline & $\begin{array}{l}\overline{\Delta f_{n}} \\
\mathrm{mHz}\end{array}$ & $\Delta f_{n} / \sigma_{f}$ & $\Delta f_{n} / \sigma_{u \mid v}$ & $\begin{array}{l}\overline{\Delta f_{n}} \\
\mathrm{mHz}\end{array}$ & $\Delta f_{n} / \sigma_{f}$ & $\Delta f_{n} / \sigma_{u \mid v}$ \\
\hline 1 & 0.00 & 0.00 & 0.00 & 0.00 & 0.00 & 0.00 \\
\hline 2 & -0.90 & -0.21 & -0.39 & -0.50 & -0.12 & -0.22 \\
\hline 3 & -0.10 & -0.04 & -0.09 & -0.20 & -0.07 & -0.18 \\
\hline 4 & -1.90 & -0.42 & -1.28 & -1.00 & -0.22 & -0.67 \\
\hline 5 & 0.00 & 0.00 & 0.00 & 0.00 & 0.00 & 0.00 \\
\hline 6 & -0.60 & -0.08 & -0.27 & -2.10 & -0.27 & -0.93 \\
\hline 7 & 0.00 & 0.00 & 0.00 & 0.00 & 0.00 & 0.00 \\
\hline 8 & -1.30 & -0.11 & -0.32 & -7.40 & -0.63 & -1.83 \\
\hline 9 & 0.00 & 0.00 & 0.00 & 0.00 & 0.00 & 0.00 \\
\hline 10 & -8.20 & -0.78 & -1.70 & -10.60 & -1.01 & -2.19 \\
\hline 11 & 0.00 & 0.00 & 0.00 & 0.00 & 0.00 & 0.00 \\
\hline 12 & -0.10 & -0.01 & -0.02 & -0.90 & -0.06 & -0.17 \\
\hline
\end{tabular}


The frequency shift was added to the last 5000 samples. The EVS control charts for the first principal component scores of the residuals with damage scenarios $D 1-D 4$ are plotted in Figures 9-12, respectively. All damage scenarios clearly show an abrupt and permanent change in the data at the end of the data record. Occasional false alarms emerged. The subgroup maxima decreased below zero causing an alarm, but the subgroup minima stayed mainly within the control limits. This is probably due to outliers in the training data. Nevertheless, the shifts in the minima can be visually observed in the figures.

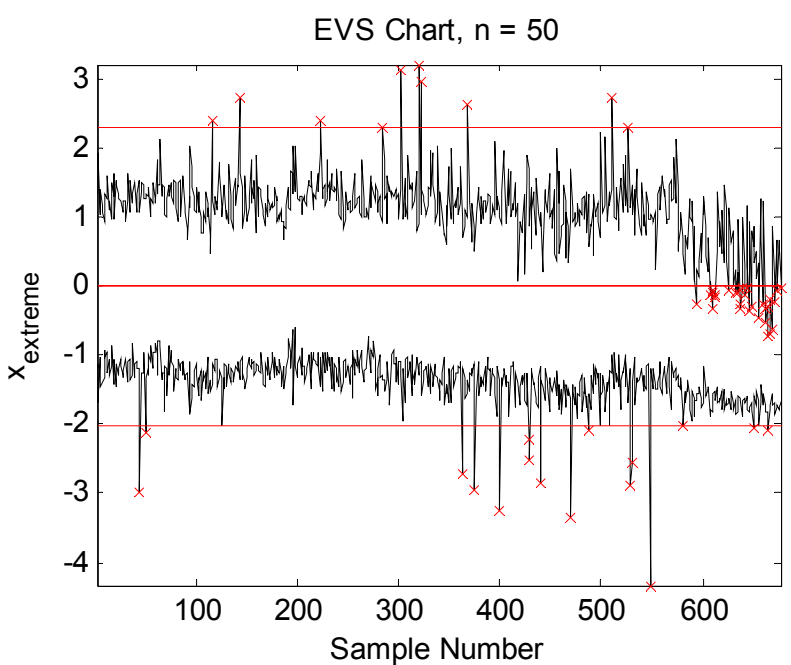

Figure 9. EVS chart of the first PC scores of the residual with damage $D 1$.

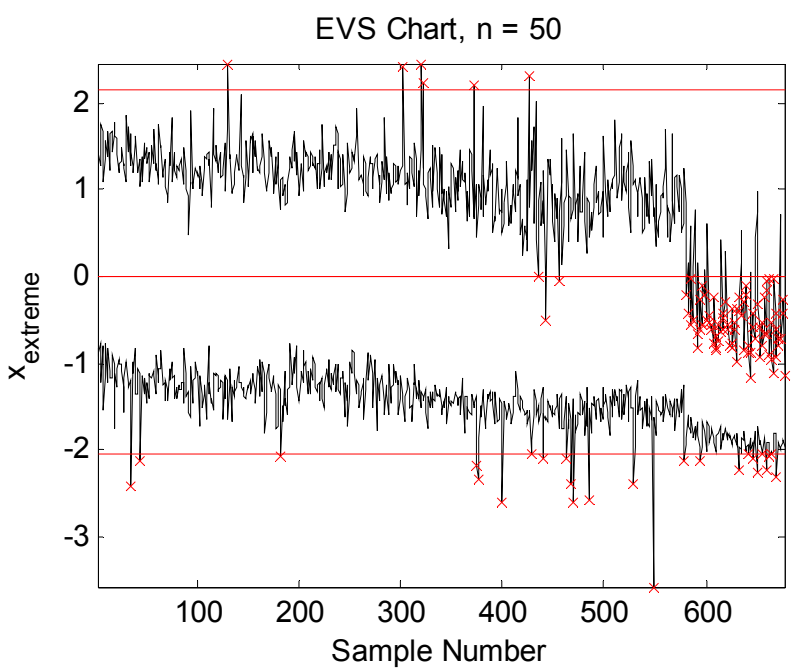

Figure 10. EVS chart of the first PC scores of the residual with damage $D 2$.

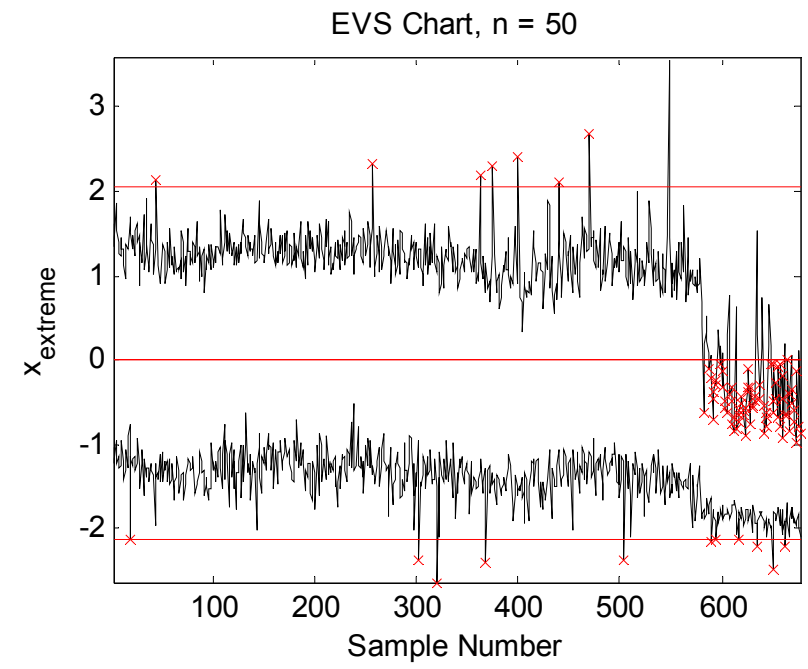

Figure 11. D3: EVS chart of the first PC scores of the residual with damage $D 3$.

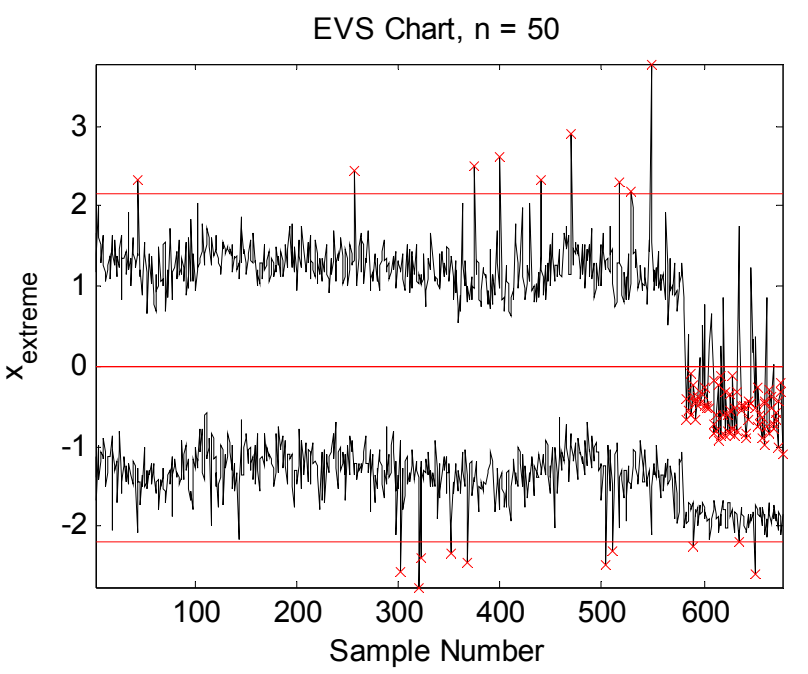

Figure 12. D4: EVS chart of the first PC scores of the residual with damage $D 4$.

\section{CONCLUSION}

A structural health monitoring system was introduced with appropriate hardware and software and applied to an arch bridge in Porto. Vibration data during two years of continuous monitoring were analysed to track changes in the identified natural frequencies of the structure.

Seasonal changes were clearly present in the natural frequencies, which made damage detection difficult. The correlation between the frequencies was utilized to remove the environmental effects from the data using the minimum mean error estimation algorithm. Measurement of the environmental variables (e.g. temperature) was not necessary.

The structure was known to be undamaged during the monitoring period, which was compatible with the results of the data analysis.

For a research study, artificial damage was simulated with frequency shifts. These shifts were detect- 
ed if environmental effects were first removed from the data but remained undetected otherwise.

The presence of outliers in the data is another concern. Using a relatively small subgroup size (50), damage was more easily detected. A small subgroup size would be more sensitive to damage but also sensitive to false alarms. A large subgroup size is quite insensitive to damage if outliers exist in the training data. Therefore, outlier detection and correction in the training data would be an important function in SHM systems.

\section{REFERENCES}

Adão da Fonseca, A. \& Millanes Mato, F. 2005. The Infante Henrique Bridge over the River Douro, in Porto, Portugal. Structural Engineering International 15.

Alampalli, S. 1998. Influence of in-service environment on modal parameters. In Proceedings of the 16th International Modal Analysis Conference, Santa Barbara, California, 25 February 1998: 111-116. Bethel, Connecticut: Society for Experimental Mechanics.

Basseville, M., Bourquin, F., Mevel, L., Nasser, H. \& Treyssède, F. 2006. Merging sensor data from multiple temperature scenarios for vibration-based monitoring of civil structures. In A. Güemes (ed), Proceedings of the third European Workshop on Structural Health Monitoring, Granada, Spain, 2006: 759-506. Granada, Spain, 5-7 July, 2006. DEStech Publications.

Bishop, C.M. 2006. Pattern recognition and machine learning. New York: Springer.

Bocca, M., Mahmood, A., Eriksson, L.M., Kullaa, J. \& Jäntti, R. 2011. A synchronized wireless sensor network for experimental modal analysis in structural health monitoring, Computer-Aided Civil and Infrastructure Engineering 26(7): 483-499.

Castillo, E. 1988. Extreme value theory in engineering. San Diego, CA: Academic Press.

Cornwell, P., Farrar, C.R., Doebling, S.W. \& Sohn, H. 1999. Structural testing series: Part 4. Environmental variability of modal properties, Experimental Techniques, 23(6): 4548.

Darbre, G.R. \& Proulx, J. 2001. The need for system identification techniques using ambient vibration data recorded on large dams. In M. Link (ed.), Proceedings of the International Conference on Structural System Identification, Kassel, Germany, 5-7 September, 2001: 205-213. University of Kassel.

Farrar, C.R., Doebling, S.W., Cornwell, P.J. \& Straser, E.G. 1997. Variability of modal parameters measured on the Alamosa Canyon Bridge. In Proceedings of IMAC-XV: $A$ Conference on Structural Dynamics, Orlando, Florida, Fevruary 1997: 257-263. Bethel, Connecticut: Society for Experimental Mechanics.

Gorinevsky, D., Kim, S.-J., Boyd, S., Gordon, G., Beard, S. \& Chang, F.-K. 2007. Optimal estimation of accumulating damage trend from series of SHM images. In F.K. Chang (ed.), Proceedings of the 6th International Workshop on Structural Health Monitoring, Stanford, CA, 11-13 September 2007: 1340-1346. Stanford University: DEStech Publications.

Kullaa, J. 2002. Elimination of environmental influences from damage-sensitive features in a structural health monitoring syste. In D.L.Balageas (ed.), Proceedings of the First European Workshop on Structural Health Monitoring, Paris, 10-12 July 2002: 742-749. Onera: DEStech Publications.
Kullaa, J. 2003. Is temperature measurement essential in structural health monitoring? In F.K. Chang (ed.), Proceedings of the 4th International Workshop on Structural Health Monitoring. Stanford, CA, 15-17 September 2003: 717724. Stanford University: DEStech Publications.

Kullaa, J. 2004. Latent variable models to eliminate the environmental effects in structural health monitoring. In $\mathrm{R}$. Flesch, H. Irschik and M. Krommer (eds.), Proceedings of the Third European Conference on Structural Control, Volume II, Vienna, Austria, 12-15 July 2004: S5-55-S5-58. Vienna University of Technology.

Kullaa, J. 2005. Damage detection under a varying environment using the missing data concept. In F.K. Chang (ed.), Proceedings of the 5th International Workshop on Structural Health Monitoring, Stanford, CA, 12-14 September 2005: 565-573. Stanford University: DEStech Publications.

Kullaa, J. 2006. Removing non-linear environmental influences from structural features. In A. Güemes (ed.), Proceedings of the third European Workshop on Structural Health Monitoring, Granada, Spain, 5-7 July 2006: 635-642. DEStech Publications.

Kullaa, J. 2010. Sensor validation using minimum mean square error estimation. Mechanical Systems and Signal Processing 24(5): 1444-1457.

Kullaa, J. 2011. Distinguishing between sensor fault, structural damage, and environmental or operational effects in structural health monitoring. Mechanical Systems and Signal Processing 25(8): 2976-2989.

Kullaa, J. 2012. Whitening transformation in damage detection. In A.E. Del Grosso, P. Basso (eds.), Smart structures: Proceedings of the 5th European Conference on Structural Control - EACS 2012, Genoa, Italy, 18-20 June 2012. Erredi Grafiche Editoriali.

Kullaa, J. 2014. Structural health monitoring under nonlinear environmental or operational influences. Shock and Vibration 2014(863494).

Lämsä, V. \& Kullaa, J. 2007. Nonlinear factor analysis in structural health monitoring to remove environmental effects. In F.K. Chang (ed.), Proceedings of the 6th International Workshop on Structural Health Monitoring, Stanford, CA, 11-13 September 2007: 1092-1099. Stanford University: DEStech Publications.

Magalhães, F., Cunha, A. \& Caetano, E. 2008. Dynamic monitoring of a long span arch bridge. Engineering Structures 30(11): 3034-3044.

Magalhães, F., Cunha, A. \& Caetano, E. 2009. Online automatic identification of the modal parameters of a long span arch bridge, Mechanical Systems and Signal Processing 23: 316-329.

Magalhães, F., Cunha, A. \& Caetano, E. 2011. Vibration based structural health monitoring of an arch bridge: From automated OMA to damage detection. Mechanical Systems and Signal Processing 28: 212-228.

Magalhães, F., Cunha, A. \& Caetano, E. 2014. Five years of continuous dynamic monitoring of Infante D. Henrique Bridge. In: A. Cunha, E. Caetano, P. Ribeiro, \& G. Müller (eds.), 9th International Conference on Structural Dynamics, EURODYN 2014, Porto, Portugal, 30 June - 2 July 2014: 2263-2270.

Manson, G. 2002. Identifying damage sensitive, environment insensitive features for damage detection. In A.W. Lees, U. Prells \& J. Norton (eds.), Proceedings of the Third International Conference on Identification in Engineering Systems, Swansea, 15-17 April 2002: 187-197. Institute of Physics Publishing.

Montgomery, D.C. 1997. Introduction to statistical quality control, 3rd edition. New York: John Wiley \& Sons.

Peeters, B. \& De Roeck, G. 2000. One year monitoring of the Z24 bridge: Environmental influences versus damage effects. In Proceedings of IMAC-XVIII: A Conference on 
Structural Dynamics, San Antonio, Texas, 7-10 February 2000: 1570-1576. Bethel, Connecticut: Society for Experimental Mechanics.

Rohrmann, R.G., Baessler, M., Said, S., Schmid, W. \& Ruecker, W.F. 2000. Structural causes of temperature affected modal data of civil structures obtained by long time monitoring. In Proceedings of IMAC-XVIII: A Conference on Structural Dynamics, San Antonio, Texas, 7-10 February 2000: 1-7. Bethel, Connecticut: Society for Experimental Mechanics.

Sohn, H., Worden, K. \& Farrar, C.R. 2003. Statistical damage classification under changing environmental and operational conditions. Journal of Intelligent Materials Systems and Structures 13(9): 561-574.

Vanlanduit, S., Parloo, E., Cauberghe, B., Guillaume, P. \& Verboven, P. 2005. A robust singular value decomposition for damage detection under changing operational conditions and structural uncertainties. Journal of Sound and Vibration 284: 1033-1050.

Wahab, M.A. \& De Roeck, G. 1997. Effect of temperature on dynamic system parameters of a highway bridge. Structural Engineering International 4: 266-270.

Worden, K., Allen, D., Sohn, H. \& Farrar, C.R. 2002. Damage detection in mechanical structures using extreme value statistics. In V.S. Rao (ed.), SPIE Proceedings, Vol. 4693, 9th Annual International Symposium on Smart Structures and Materials, San Diego, CA, 2002: 289-299.

Yan, A.-M., Kerschen, G., De Boe, P. \& Golinval, J.-C. 2005a. Structural damage diagnosis under varying environmental conditions-Part I: A linear analysis. Mechanical Systems and Signal Processing 19: 847-864.

Yan, A.-M., Kerschen, G., De Boe, P. \& Golinval, J.-C. 2005 b. Structural damage diagnosis under varying environmental conditions-Part II: local PCA for non-linear cases. Mechanical Systems and Signal Processing 19: 865-880.

Yan, A.-M. \& Golinval, J.-C. 2006. Null subspace-based damage detection of structures using vibration measurements. Mechanical Systems and Signal Processing 20: 611-626. 